\title{
Body mass index and in-hospital mortality in patients with acute exacerbation of idiopathic pulmonary fibrosis
}

\author{
Nobuyasu Awano (101, Taisuke Jo (10) ${ }^{2,3}$, Hideo Yasunaga ${ }^{4}$, Minoru Inomata (1) ${ }^{1}$, \\ Naoyuki Kuse ${ }^{1}$, Mari Tone ${ }^{1}$, Kojiro Morita ${ }^{4,5}$, Hiroki Matsui ${ }^{4}$, Kiyohide Fushimi ${ }^{6}$, \\ Takahide Nagase $^{3}$ and Takehiro Izumo ${ }^{1}$
}

Affiliations: ${ }^{1}$ Dept of Respiratory Medicine, Japanese Red Cross Medical Center, Tokyo, Japan. ${ }^{2}$ Dept of Health Services Research, Graduate School of Medicine, The University of Tokyo, Tokyo, Japan. ${ }^{3}$ Dept of Respiratory Medicine, Graduate School of Medicine, The University of Tokyo, Tokyo, Japan. ${ }^{4}$ Dept of Clinical Epidemiology and Health Economics, School of Public Health, The University of Tokyo, Tokyo, Japan. ${ }^{5}$ Dept of Health Services Research, Faculty of Medicine, University of Tsukuba, Ibaraki, Japan. ${ }^{6}$ Dept of Health Policy and Informatics, Tokyo Medical and Dental University Graduate School of Medicine, Tokyo, Japan.

Correspondence: Nobuyasu Awano, Dept of Respiratory Medicine, Japanese Red Cross Medical Center, 4-1-22 Hiroo, Shibuya-ku, Tokyo 150-8935, Japan. E-mail: awanobu0606ahotmail.co.jp

\section{ABSTRACT}

Background: Idiopathic pulmonary fibrosis (IPF) is an interstitial lung disease characterised by chronic fibrosis, and acute exacerbation of IPF (AE-IPF) is the leading cause of death in patients with IPF. Data on the association between the body mass index (BMI) and prognosis of AE-IPF are lacking. This study was performed to evaluate the association between BMI and in-hospital mortality in patients who developed AE-IPF using a national inpatient database.

Methods: Using the Japanese Diagnosis Procedure Combination database, we retrospectively collected data of inpatients with AE-IPF from 1 July, 2010 to 31 March, 2018. We performed a multivariable logistic regression analysis to evaluate the association between all-cause in-hospital mortality and BMI, categorised as underweight $\left(<18.5 \mathrm{~kg} \cdot \mathrm{m}^{-2}\right)$, low-normal weight $\left(18.5-22.9 \mathrm{~kg} \cdot \mathrm{m}^{-2}\right)$, high-normal weight $\left(23.0-24.9 \mathrm{~kg} \cdot \mathrm{m}^{-2}\right)$, overweight $\left(25.0-29.9 \mathrm{~kg} \cdot \mathrm{m}^{-2}\right)$ and obese $\left(\geqslant 30.0 \mathrm{~kg} \cdot \mathrm{m}^{-2}\right)$.

Results: In total, 14783 patients were eligible for this study. The in-hospital mortality rate was 59.0\%, 55.0\%, $53.8 \%, 54.8 \%$ and $46.0 \%$ in the underweight, low-normal weight, high-normal weight, overweight and obese groups, respectively. Underweight patients had a significantly higher mortality rate (OR 1.25, 95\% CI $1.10-1.42$ ) and obese patients had a significantly lower mortality rate (OR $0.71,95 \%$ CI $0.54-0.94$ ) than lownormal weight patients.

Conclusion: Among patients with AE-IPF, the underweight group had higher mortality and the obese group had lower mortality.

@ERSpublications

Among patients with acute exacerbation of idiopathic pulmonary fibrosis, underweight patients have higher mortality and obese patients lower mortality https://bit.ly/3eoVMOR

Cite this article as: Awano N, Jo T, Yasunaga H, et al. Body mass index and in-hospital mortality in patients with acute exacerbation of idiopathic pulmonary fibrosis. ERJ Open Res 2021; 7: 00037-2021 [https://doi.org/10.1183/23120541.00037-2021].

Received: 19 Jan 2021 | Accepted: 12 April 2021

Copyright $\odot$ The authors 2021. This version is distributed under the terms of the Creative Commons Attribution NonCommercial Licence 4.0. For commercial reproduction rights and permissions contact permissions@ersnet.org 


\section{Introduction}

Patients with idiopathic pulmonary fibrosis (IPF), an interstitial lung disease characterised by chronic fibrosis, have a poor prognosis with an average survival time of 3 to 4 years [1]. A previous study showed that acute exacerbation of IPF (AE-IPF) was associated with high mortality with a mean survival time of $<1$ year and a 90-day mortality rate of $\sim 50 \%$ after AE-IPF [2]. Risk factors for AE-IPF include oxygen administration, use of antacids, smoking, low lung function, a high serum Krebs von den Lungen-6 concentration, secondary pulmonary hypertension and seasonality [3-5].

Generally, undernutrition is a potential prognostic factor in patients with respiratory diseases such as COPD [6] and pulmonary tuberculosis [7]. Moreover, protective effects of adipose tissue, referred to as the "obesity paradox", are known in many chronic diseases including cardiovascular disease [8], chronic heart failure [9] and COPD [10]. In one study of patients with IPF, one-third of the patients were undernourished [11], and a lower body mass index (BMI) at the time of diagnosis has been proposed as a prognostic factor [12-16]. To the best of our knowledge, however, no study has focused on the association between BMI and prognosis of AE-IPF.

The present study was performed using a nationwide inpatient database to evaluate the association between BMI and in-hospital mortality in patients who developed AE-IPF.

\section{Patients and methods}

\section{Data source}

Inpatient data were extracted from the Japanese Diagnosis Procedure Combination database, the details of which have been reported elsewhere [17]. More than 1000 hospitals voluntarily contribute to the database, representing $\sim 50 \%$ of all discharges from acute care hospitals in Japan. The data used in the present study included sex and age; body weight and height; smoking index; severity of dyspnoea based on the Hugh-Jones dyspnoea scale [18]; consciousness level on admission; intensive care unit (ICU) and/or emergency ward admission during hospitalisation; dates of hospitalisation and discharge; main diagnoses and pre-existing comorbidities on admission recoded by the attending physicians with the International Classification of Diseases, 10th revision (ICD-10) codes accompanied by text in Japanese; surgical and nonsurgical procedures and dates of the procedures performed; dates and doses of drugs administered during hospitalisation; and discharge status.

The Institutional Review Board of The University of Tokyo approved this study. The requirement for informed consent was waived because of the anonymous nature of the data.

\section{Patient selection}

This study used data from July 1, 2010 to March 31, 2018. The inclusion criteria were an age of $\geqslant 15$ years, diagnosis of interstitial pneumonia (ICD-10 codes J84.1, J84.8 and J84.9), examination by computed tomography within 1 day after admission, and treatment with methylprednisolone at 500 to $1000 \mathrm{mg} \cdot \mathrm{day}^{-1}$ intravenously for 3 days starting within 4 days after admission [19]. Patients with IPF were selected as follows. First, patients with idiopathic interstitial pneumonias (IIPs) other than IPF, such as idiopathic nonspecific interstitial pneumonia, respiratory bronchiolitis-associated interstitial lung disease, cryptogenic organising pneumonia, acute interstitial pneumonia, desquamative interstitial pneumonia, lymphoid interstitial pneumonia, idiopathic pleuroparenchymal fibroelastosis and unclassifiable idiopathic interstitial pneumonia, were excluded using the diagnoses in Japanese. Then, we excluded patients with the following secondary interstitial lung diseases identified using ICD-10 codes: hypersensitivity pneumonitis (J67), connective tissue disease associated with interstitial lung disease (M05, M06 and M30-35), sarcoidosis (D86), amyloidosis (E85), drug-induced lung disease (J70), radiation pneumonitis (J70), Pneumocystis jirovecii pneumonia (B59), pneumoconiosis (J60-65), pulmonary alveolar proteinosis ( J84.0), eosinophilic pneumonia (J82), Langerhans cell histiocytosis (C96) and lymphangioleiomyomatosis (D21.9). We then excluded patients who received any of the following medications related to acute heart failure within 1 day after admission: furosemide, azosemide, carperitide, landiolol hydrochloride, digoxin, deslanoside and tolvaptan [20]. We also excluded patients who underwent intra-aortic balloon pump therapy during hospitalisation. The remaining patients were assumed to have IPF. Finally, we excluded patients with missing data regarding consciousness and those who died within 4 days after admission.

\section{Patient characteristics and BMI categories}

The patient characteristics evaluated in this study were BMI; age; sex; Hugh-Jones dyspnoea scale class on admission; consciousness on admission; smoking index; comorbidities; Charlson comorbidity index; surgical and nonsurgical procedures including tracheostomy, mechanical ventilation and use of medications for IPF during hospitalisation; and continuous renal replacement therapy within 1 day after admission. Consciousness on admission was evaluated using the Japan Coma Scale [21, 22], which is 
widely used in Japan and has been shown to be well correlated with the Glasgow Coma Scale assessment [23]. The following comorbidities were identified using ICD-10 codes: lung cancer (C34), COPD (J44), pneumonia (J18), aspiration pneumonia (J69), pulmonary embolism (I26), chronic heart failure (I50), chronic renal failure (N18) and diabetes mellitus (E11). The Charlson comorbidity index was classified into five groups: $0,1,2,3-5$ and $\geqslant 6$.

BMI categories were assigned based on the World Health Organization classifications of underweight $\left(<18.5 \mathrm{~kg} \cdot \mathrm{m}^{-2}\right)$, normal weight $\left(18.5-24.9 \mathrm{~kg} \cdot \mathrm{m}^{-2}\right)$, overweight $\left(25.0-29.9 \mathrm{~kg} \cdot \mathrm{m}^{-2}\right)$ and obese $\left(\geqslant 30.0 \mathrm{~kg} \cdot \mathrm{m}^{-2}\right)$ individuals. Normal weight was further divided into low-normal $\left(18.5-22.9 \mathrm{~kg} \cdot \mathrm{m}^{-2}\right)$ and high-normal $\left(23.0-24.9 \mathrm{~kg} \cdot \mathrm{m}^{-2}\right)[24,25]$.

\section{Outcome}

The primary outcome was all-cause in-hospital mortality.

\section{Statistical analysis}

Continuous variables are presented as mean \pm standard deviation or median (interquartile range). The Kruskal-Wallis test was used to compare these variables between the groups. Proportions of categorical variables were compared using the Chi-squared test.

Missing data were observed for age, BMI, Hugh-Jones dyspnoea scale class and smoking index. First, we performed a multiple imputation procedure to replace each missing value with a set of submitted plausible values using a Markov chain Monte Carlo algorithm known as imputation by chained equations [26], thereby creating 20 filled-in complete datasets. The multiple imputation method assumes that data are missing at random and that any systemic differences between the missing and observed values can be explained by differences in the observed data $[27,28]$. We then performed multivariable logistic regression analyses fitted with generalised estimating equations to estimate the odds ratio of in-hospital mortality for each BMI category. We defined the low-normal weight group as the reference category. Finally, the results of the multivariable logistic regression analyses from the 20 datasets were combined using Rubin's rule.

Secondly, we conducted a complete-case analysis that excluded all patients with missing data. Multivariable logistic regression analysis for in-hospital mortality was performed to estimate the odds ratio for each BMI category with adjustment for other patient background factors while also adjusting for within-hospital clustering by means of a generalised estimating equation [29].

The threshold for significance was $\mathrm{p}<0.05$. All statistical analyses were performed using STATA/MP version 16 software (STATA Corp., College Station, TX, USA).

\section{Results}

During the study period, 95221 patients underwent computed tomography within 1 day after admission and received high-dose methylprednisolone for 3 days starting within 4 days after admission (figure 1).

Patients with interstitial pneumonia receiving computed tomography within 1 day after admission and receiving high-dose methylprednisolone within 4 days after admission $\mathrm{n}=95221$

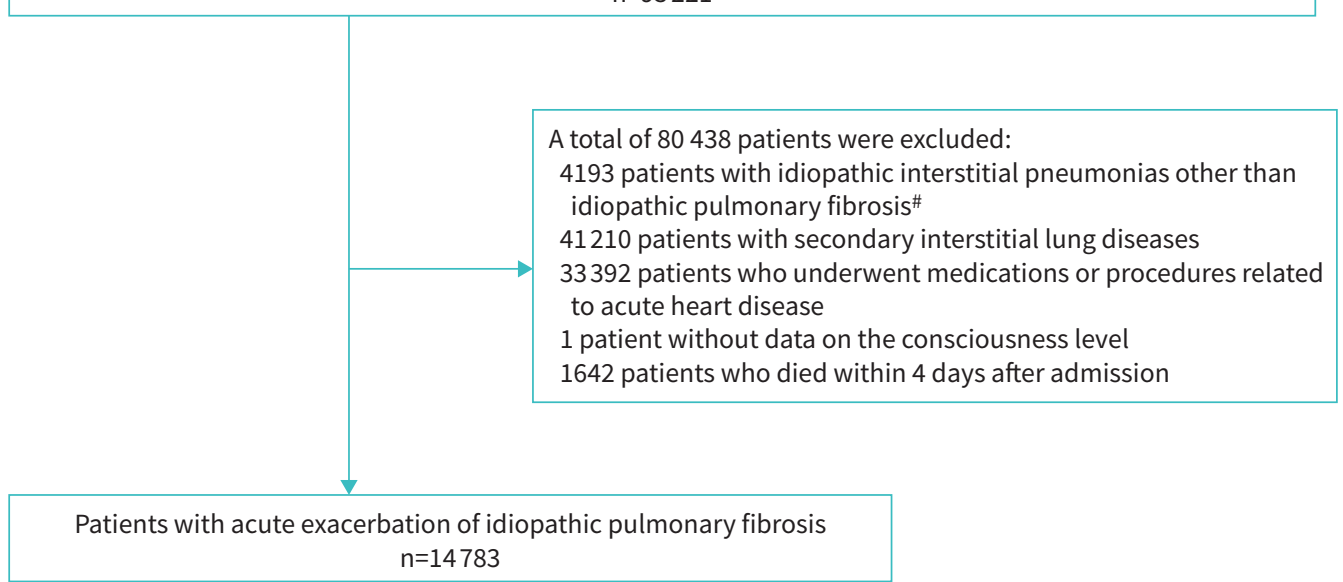

FIGURE 1 Flow chart of patient selection. \#: idiopathic nonspecific interstitial pneumonia, respiratory bronchiolitis-associated interstitial lung disease, cryptogenic organising pneumonia, acute interstitial pneumonia, desquamative interstitial pneumonia, lymphoid interstitial pneumonia, idiopathic pleuroparenchymal fibro-elastosis and unclassifiable idiopathic interstitial pneumonia. 
Among these 95221 patients, 14783 were eligible for this study. Their mean age was $75.0 \pm 9.7$ years, and the proportion of men was $71.7 \%(\mathrm{n}=10594)$. Their mean BMI was $22.4 \pm 3.7 \mathrm{~kg} \cdot \mathrm{m}^{-2}$, and $8294(56.1 \%)$ patients died during hospitalisation. The proportions of patients with missing data for age, BMI, Hugh-Jones dyspnoea scale class and smoking index were $0.6 \%(n=89), 11.0 \%(n=1629), 22.7 \%(n=3359)$ and $12.4 \%(\mathrm{n}=1830)$ of all eligible patients, respectively.

The patient characteristics for each BMI category are shown in table 1. The proportion of patients aged $>80$ years was higher in the underweight group but lower in the obese group. The proportion of females was higher in the underweight and obese groups. The proportion of patients with a poor level of consciousness on admission was higher in the underweight group than in the other groups. The proportion of patients with a Charlson comorbidity index of $\geqslant 6$ was higher in the lower BMI groups. However, the obese group had the highest percentage of patients admitted to the ICU. The percentages of lung cancer and chronic renal failure were higher in the lower BMI categories. Conversely, the percentage of diabetes mellitus was higher in the higher BMI categories. The percentages of the following treatments and procedures were higher in the higher BMI categories: azithromycin, sulfamethoxazole trimethoprim, intravenous cyclophosphamide, cyclosporin, tacrolimus, pirfenidone, nintedanib, sivelestat sodium hydrate and mechanical ventilation.

Figure 2 shows the all-cause in-hospital mortality rate for each BMI category. The in-hospital mortality rate was $59.0 \%, 55.0 \%, 53.8 \%, 54.8 \%$ and $46.0 \%$ in the underweight, low-normal weight, high-normal weight, overweight and obese groups, respectively.

Table 2 shows the results of the multivariable logistic regression analysis for all-cause in-hospital mortality using the multiple imputation method for missing data. The mortality rate in the underweight group was significantly higher than that in the reference low-normal weight group (OR 1.25, 95\% CI 1.10-1.42). In contrast, the mortality rate in the obese group was significantly lower than that in the reference low-normal weight group (OR 0.71, 95\% CI 0.54-0.94). Older age, male sex, more severe dyspnoea scores and a higher Charlson comorbidity index were significantly associated with higher mortality. In contrast, ICU admission, emergency unit admission and care at an academic hospital were associated with lower mortality. With respect to comorbidities, lung cancer and chronic renal failure were associated with higher mortality, whereas COPD was associated with lower mortality. The following treatments and procedures were associated with higher mortality: intravenous or oral cyclophosphamide, cyclosporin, azathioprine, sivelestat sodium hydrate, thrombomodulin $\alpha$, mechanical ventilation and tracheotomy. In contrast, azithromycin and sulfamethoxazole trimethoprim were associated with lower mortality.

In the complete-case multivariable logistic regression analysis, the OR (95\% CI) with reference to the low-normal weight group were 1.25 (1.06-1.46), 0.94 (0.83-1.07), $1.01(0.90-1.15)$ and $0.75(0.54-0.94)$ for the underweight, high-normal weight, overweight and obese groups, respectively.

\section{Discussion}

Using a nationwide inpatient database in Japan, we investigated the association between BMI and mortality in patients with AE-IPF. Patients in the underweight group had a significantly higher mortality rate and those in the obese group had a significantly lower mortality rate than patients in the other weight groups. To our knowledge, the present study is the first to demonstrate a relationship between BMI and mortality in patients with AE-IPF.

Studies have been performed to evaluate the relationship between patients with IPF and body weight. A previous study showed that patients who lost $\geqslant 5 \%$ of body weight during the first year after diagnosis of IPF had a poorer prognosis than those who did not [12]. Moreover, staging based on annual body weight loss is reportedly a useful predictor of the prognosis of IPF [16]. These studies have suggested a detrimental impact of a lower BMI on patients with IPF, whereas other studies have, although indirectly, depicted a detrimental impact of obesity on patients with IPF. For example, one study showed that a decline in the forced vital capacity was a prognostic factor for patients with IPF [30], but others showed that an increased BMI was associated with lower vital capacity [31] and forced vital capacity [32] in the general population. Data regarding the impact of BMI on AE-IPF are inconsistent. One report indicated that BMI was not a risk factor for developing acute exacerbation [4], whereas another study showed that high BMI was a risk factor for developing acute exacerbation [33]. To our knowledge, however, no previous study has examined the relationship between BMI and mortality in patients with AE-IPF. The in-hospital mortality rate for all patients with AE-IPF in the current study was $56.1 \%$, which is similar to previously reported rates [2]. The underweight group had the highest mortality rate, and the obese group had the lowest. A British database study demonstrated that the association between BMI and mortality varied among diseases [34]. Some diseases had a J-shaped association with BMI and other diseases had an inverse linear association with BMI. The results of our study were similar to the association between BMI 
TABLE 1 Patients' characteristics and comorbidities in relation to body mass index (BMI) category

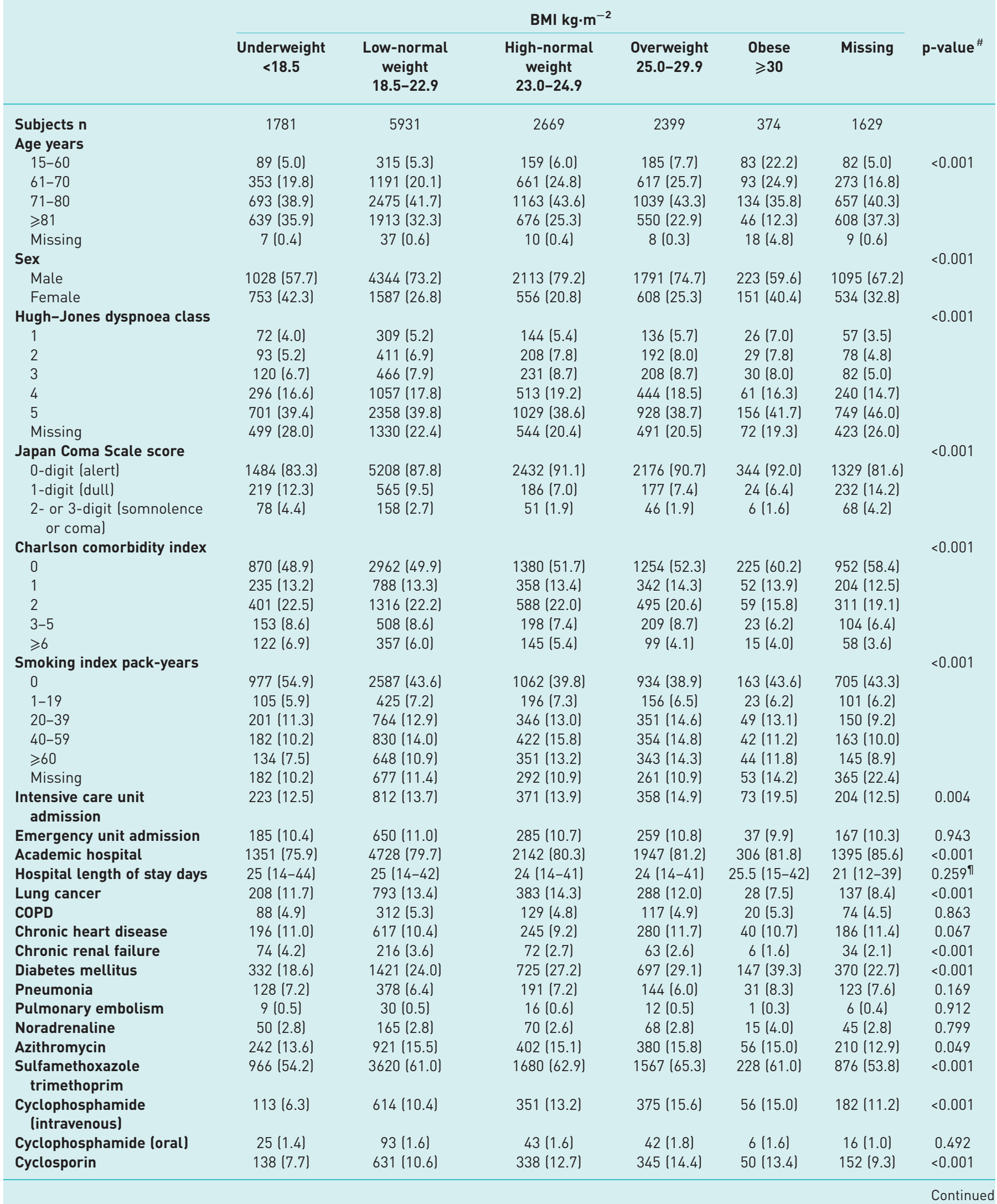




\section{TABLE 1 Continued}

\begin{tabular}{|c|c|c|c|c|c|c|c|}
\hline & \multicolumn{6}{|c|}{ BMI $\mathrm{kg} \cdot \mathrm{m}^{-2}$} & p-value \\
\hline Tacrolimus & $26(1.5)$ & 115 (1.9) & 49 (1.8) & $64(2.7)$ & 12 (3.2) & $15(0.9)$ & 0.001 \\
\hline Azathioprine & $18(1.0)$ & $101(1.7)$ & $57(2.1)$ & $46(1.9)$ & $5(1.3)$ & $25(1.5)$ & 0.097 \\
\hline Pirfenidone & $68(3.8)$ & $265(4.5)$ & $111(4.2)$ & $152(6.3)$ & $23(6.1)$ & $59(3.6)$ & $<0.001$ \\
\hline Thrombomodulin $\alpha$ & $106(6.0)$ & $357(6.0)$ & $170(6.4)$ & $157(6.5)$ & $26(7.0)$ & 95 (5.8) & 0.868 \\
\hline Mechanical ventilation & $425(23.9)$ & 1619 (27.3) & $787(29.5)$ & 751 (31.3) & 134 (35.8) & 500 (30.7) & $<0.001$ \\
\hline Haemodialysis & $31(1.7)$ & $82(1.4)$ & $28(1.0)$ & $28(1.2)$ & $3(0.8)$ & 19 (1.2) & 0.344 \\
\hline Tracheotomy & $57(3.2)$ & $183(3.1)$ & 86 (3.2) & $67(2.8)$ & $19(5.1)$ & $47(2.9)$ & 0.299 \\
\hline
\end{tabular}

and mortality of lung cancer in that study. Obesity may be a risk factor for developing AE-IPF, but it may be favourable in patients who developed AE-IPF. The mechanism by which obese patients with AE-IPF have favourable outcomes remains unknown.

BMI can be influenced by a patient's background factors, such as ethnic characteristics. Reports have suggested that Asian ethnic populations have different associations between BMI and health risks than Western populations [35]. Additionally, Asian ethnic populations generally have a higher percentage of body fat than Caucasians of the same age, sex and BMI, which may contribute to the difference in the properties of fat, including adipocytokines such as adiponectin, leptin and resistin [35, 36]. The BMI of patients with IPF in the present Japanese study was lower than that reported from other countries [14]. Such a difference in BMI distribution between Asian and Caucasian patients with IPF has been observed in previous studies $[15,37]$. The association between BMI and prognosis in patients with AE-IPF may therefore vary among different ethnic groups.

Several limitations of this study should be acknowledged. Because the database does not include data on laboratory examinations, pulmonary function tests, performance status and radiological findings, the diagnosis and severity of IPF could not be precisely evaluated in this study. Additionally, the accuracy of the IPF diagnosis was not confirmed by radiological and pathological analyses because we based the diagnosis on physician-diagnosed IPF. To classify IPF, all cases of IIPs other than IPF and secondary interstitial pneumonia were excluded using the diagnoses in Japanese or ICD-10 codes, because the specificity of diagnoses in the Diagnosis Procedure Combination (DPC) data are high in general [38].

In conclusion, this study has demonstrated that the underweight group had higher mortality and the obese group had lower mortality in patients with AE-IPF.

FIGURE 2 All-cause in-hospital mortality in patients with acute exacerbation of idiopathic pulmonary fibrosis in relation to body mass index category.

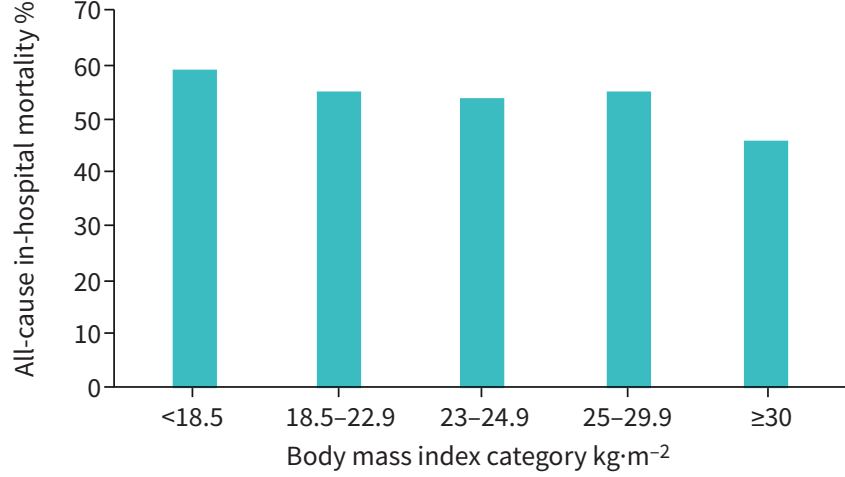




\section{TABLE 2 Multivariable logistic regression analysis for all-cause in-hospital mortality}

\begin{tabular}{|c|c|c|c|}
\hline & Adjusted OR & $95 \% \mathrm{Cl}$ & p-value \\
\hline \multicolumn{4}{|l|}{ Body mass index $\mathrm{kg} \cdot \mathrm{m}^{-2}$} \\
\hline$<18.5$ & 1.25 & $1.10-1.42$ & 0.001 \\
\hline $18.5-22.9$ & Reference & & \\
\hline $23.0-24.9$ & 0.92 & $0.82-1.02$ & 0.122 \\
\hline $25.0-29.9$ & 0.98 & $0.88-1.09$ & 0.706 \\
\hline$\geqslant 30.0$ & 0.71 & $0.54-0.94$ & 0.016 \\
\hline \multicolumn{4}{|l|}{ Age years } \\
\hline $15-60$ & Reference & & \\
\hline $61-70$ & 1.86 & $1.55-2.29$ & $<0.001$ \\
\hline $71-80$ & 2.32 & $1.94-2.77$ & $<0.001$ \\
\hline$\geqslant 81$ & 2.98 & $2.47-3.59$ & $<0.001$ \\
\hline \multicolumn{4}{|l|}{ Sex } \\
\hline Female & Reference & & \\
\hline Male & 1.37 & $1.23-1.53$ & $<0.001$ \\
\hline \multicolumn{4}{|l|}{ Hugh-Jones dyspnoea class } \\
\hline 1 & Reference & & \\
\hline 2 & 1.20 & $0.94-1.52$ & 0.137 \\
\hline 3 & 1.41 & $1.12-1.78$ & 0.003 \\
\hline 4 & 2.02 & $1.62-2.51$ & $<0.001$ \\
\hline 5 & 4.91 & $4.02-6.01$ & $<0.001$ \\
\hline \multicolumn{4}{|l|}{ Japan Coma Scale score } \\
\hline 0-digit (alert) & Reference & & \\
\hline 1-digit (dull) & 1.19 & $1.02-1.39$ & 0.024 \\
\hline 2- or 3-digit (somnolence or coma) & 0.97 & $0.73-1.29$ & 0.822 \\
\hline \multicolumn{4}{|l|}{ Charlson comorbidity index } \\
\hline 0 & Reference & & \\
\hline 1 & 0.87 & $0.77-0.98$ & 0.027 \\
\hline 2 & 1.23 & $1.09-1.38$ & 0.001 \\
\hline $3-5$ & 1.06 & $0.89-1.28$ & 0.503 \\
\hline$\geqslant 6$ & 2.60 & $2.12-3.19$ & $<0.001$ \\
\hline \multicolumn{4}{|l|}{ Smoking index pack-years } \\
\hline 0 & Reference & & \\
\hline $1-19$ & 0.84 & $0.72-0.98$ & 0.026 \\
\hline $20-39$ & 0.82 & $0.73-0.93$ & 0.002 \\
\hline $40-59$ & 0.77 & $0.67-0.89$ & $<0.001$ \\
\hline$\geqslant 60$ & 0.78 & $0.68-0.90$ & 0.001 \\
\hline Intensive care unit admission & 0.78 & $0.67-0.91$ & 0.001 \\
\hline Emergency unit admission & 0.79 & $0.67-0.94$ & 0.007 \\
\hline Academic hospital & 0.70 & $0.63-0.78$ & $<0.001$ \\
\hline Lung cancer & 2.28 & $1.94-2.69$ & $<0.001$ \\
\hline COPD & 0.77 & $0.63-0.94$ & 0.010 \\
\hline Chronic heart disease & 1.05 & $0.91-1.22$ & 0.484 \\
\hline Chronic renal failure & 1.65 & $1.26-2.16$ & $<0.001$ \\
\hline Diabetes mellitus & 0.94 & $0.85-1.03$ & 0.177 \\
\hline Pneumonia & 0.97 & $0.81-1.16$ & 0.724 \\
\hline Pulmonary embolism & 0.83 & $0.48-1.45$ & 0.516 \\
\hline Noradrenaline & 0.80 & $0.62-1.03$ & 0.084 \\
\hline Azithromycin & 0.79 & $0.69-0.89$ & 0.001 \\
\hline Sulfamethoxazole trimethoprim & 0.42 & $0.39-0.46$ & $<0.001$ \\
\hline Cyclophosphamide (intravenous) & 4.20 & $3.59-4.91$ & $<0.001$ \\
\hline Cyclophosphamide (oral) & 2.84 & $1.99-4.06$ & $<0.001$ \\
\hline Cyclosporin & 2.31 & $1.99-2.68$ & $<0.001$ \\
\hline Tacrolimus & 0.89 & $0.64-1.22$ & 0.688 \\
\hline Azathioprine & 1.77 & $1.21-2.60$ & 0.003 \\
\hline Pirfenidone & 1.13 & $0.93-1.39$ & 0.223 \\
\hline Nintedanib & 0.76 & $0.57-1.01$ & 0.063 \\
\hline Sivelestat sodium hydrate & 1.33 & $1.13-1.55$ & $<0.001$ \\
\hline Thrombomodulin $\alpha$ & 3.07 & $2.35-4.01$ & $<0.001$ \\
\hline Mechanical ventilation & 4.01 & $3.54-4.53$ & $<0.001$ \\
\hline Haemodialysis & 1.26 & $0.81-1.96$ & 0.305 \\
\hline Tracheotomy & 1.82 & $1.30-2.54$ & $<0.001$ \\
\hline
\end{tabular}


Author contributions: N. Awano designed the study, analysed and interpreted the data, and prepared the manuscript. T. Jo designed the study, analysed and interpreted the data, and prepared the manuscript. H. Yasunaga analysed and interpreted the data and prepared the manuscript. M. Inomata interpreted the data. N. Kuse interpreted the data. M. Tone interpreted the data. K. Morita collected and interpreted the data. H. Matsui collected the data. K. Fushimi collected the data. T. Nagase interpreted the data and prepared the manuscript. T. Izumo interpreted the data and prepared the manuscript. All authors approved the final manuscript.

Conflict of interest: N. Awano has nothing to disclose. T. Jo has nothing to disclose. H. Yasunaga reports grants from The Ministry of Health, Labour and Welfare, Japan, and The Ministry of Education, Culture, Sports, Science and Technology, Japan, during the conduct of the study. M. Inomata has nothing to disclose. N. Kuse has nothing to disclose. M. Tone has nothing to disclose. K. Morita has nothing to disclose. H. Matsui has nothing to disclose. K. Fushimi has nothing to disclose. T. Nagase has nothing to disclose. T. Izumo has nothing to disclose.

Support statement: This work was supported by grants from the Ministry of Health, Labour and Welfare, Japan (19AA2007 and H30-Policy-Designated-004), and a Grant-in-Aid for Scientific Research from the Ministry of Education, Culture, Sports, Science and Technology, Japan (17H04141). The funding bodies had no role in the design of the study; collection, analysis, or interpretation of the data; or writing of the manuscript. Funding information for this article has been deposited with the Crossref Funder Registry.

\section{References}

1 Raghu G, Collard HR, Egan JJ, et al. An official ATS/ERS/JRS/ALAT statement: idiopathic pulmonary fibrosis: evidence-based guidelines for diagnosis and management. Am J Respir Crit Care Med 2011; 183: 788-824.

2 Natsuizaka M, Chiba H, Kuronuma K, et al. Epidemiologic survey of Japanese patients with idiopathic pulmonary fibrosis and investigation of ethnic differences. Am J Respir Crit Care Med 2014; 190: 773-779.

3 Collard HR, Richeldi L, Kim DS, et al. Acute exacerbations in the INPULSIS trials of nintedanib in idiopathic pulmonary fibrosis. Eur Respir J 2017; 49: 1601339.

4 Qiu M, Chen Y, Ye Q. Risk factors for acute exacerbation of idiopathic pulmonary fibrosis: a systematic review and meta-analysis. Clin Respir J 2018; 12: 1084-1092.

5 Simon-Blancal V, Freynet O, Nunes $\mathrm{H}$, et al. Acute exacerbation of idiopathic pulmonary fibrosis: outcome and prognostic factors. Respiration 2012; 83: 28-35.

6 Landbo C, Prescott E, Lange P, et al. Prognostic value of nutritional status in chronic obstructive pulmonary disease. Am J Respir Crit Care Med 1999; 160: 1856-1861.

7 Miyata S, Tanaka M, Ihaku D. The prognostic significance of nutritional status using malnutrition universal screening tool in patients with pulmonary tuberculosis. Nutr J 2013; 12: 42.

8 Romero-Corral A, Montori VM, Somers VK, et al. Association of bodyweight with total mortality and with cardiovascular events in coronary artery disease: a systematic review of cohort studies. Lancet 2006; 368: 666-678.

9 Oreopoulos A, Padwal R, Kalantar-Zadeh K, et al. Body mass index and mortality in heart failure: a meta-analysis. Am Heart J 2008; 156: 13-22.

10 Yamauchi Y, Hasegawa W, Yasunaga H, et al. Paradoxical association between body mass index and in-hospital mortality in elderly patients with chronic obstructive pulmonary disease in Japan. Int J Chron Obstruct Pulmon Dis 2014; 9: 1337-1346.

11 Jouneau S, Kerjouan M, Rousseau C, et al. What are the best indicators to assess malnutrition in idiopathic pulmonary fibrosis patients? A cross-sectional study in a referral center. Nutrition 2019; 62: 115-121.

12 Nakatsuka Y, Handa T, Kokosi M, et al. The clinical significance of body weight loss in idiopathic pulmonary fibrosis patients. Respiration 2018; 96: 338-347.

13 Kim JH, Lee JH, Ryu YJ, et al. Clinical predictors of survival in idiopathic pulmonary fibrosis. Tuberc Respir Dis (Seoul) 2012; 73: 162-168.

14 Alakhras M, Decker PA, Nadrous HF, et al. Body mass index and mortality in patients with idiopathic pulmonary fibrosis. Chest 2007; 131: 1448-1453.

15 Mura M, Porretta MA, Bargagli E, et al. Predicting survival in newly diagnosed idiopathic pulmonary fibrosis: a 3-year prospective study. Eur Respir J 2012; 40: 101-109.

16 Kishaba T, Nagano H, Nei Y, et al. Body mass index-percent forced vital capacity-respiratory hospitalization: new staging for idiopathic pulmonary fibrosis patients. J Thorac Dis 2016; 8: 3596-3604.

17 Yasunaga H. Real World Data in Japan: Chapter II the diagnosis procedure combination database. Ann Clin Epidemiol 2019; 1: 76-79.

18 Hugh-Jones P, Lambert AV. A simple standard exercise test and its use for measuring exertion dyspnoea. $\mathrm{Br} \mathrm{Med} \mathrm{J}$ 1952; 1: 65-71.

19 Aso S, Matsui H, Fushimi K, et al. Effect of cyclosporine A on mortality after acute exacerbation of idiopathic pulmonary fibrosis. $J$ Thorac Dis 2018; 10: 5275-5282.

20 Collard HR, Ryerson CJ, Corte TJ, et al. Acute exacerbation of idiopathic pulmonary fibrosis an international working group report. Am J Respir Crit Care Med 2016; 194: 265-275.

21 Ohta T, Waga S, Hajime H, et al. New grading of level of disordered consciousness (author's translation). No Shinkei Geka 1974; 2: 623-627.

22 Shigematsu K, Nakano H, Watanabe Y. The eye response test alone is sufficient to predict stroke outcome reintroduction of Japan Coma Scale: a cohort study. BMJ Open 2013; 3: e002736.

23 Ono K, Wada K, Takahara $\mathrm{T}$, et al. Indications for computed tomography in patients with mild head injury. Neurol Med Chir (Tokyo) 2007; 47: 291-297.

24 World Health Organization. Global Database on Body Mass Index. World Health Organization; 2014. www.who. int/nutrition/databases/bmi/en/ Date last accessed: 31 October 2020.

25 Hasegawa W, Yamauchi Y, Yasunaga $\mathrm{H}$, et al. Factors affecting mortality following emergency admission for chronic obstructive pulmonary disease. BMC Pulm Med 2014; 14: 151.

26 Rubin DB, Schenker N. Multiple imputation in healthcare databases: an overview and some applications. Stat Med 1991; 10: 585-598. 

research: potential and pitfalls. BMJ 2009; 338: b2393. Li P, Stuart EA, Allison DB. Multiple imputation: a flexible tool for handling missing data. JAMA 2015; 314 : 1966-1967.

29 Hubbard AE, Ahern J, Fleischer NL, et al. To GEE or not to GEE: comparing population average and mixed models for estimating the associations between neighborhood risk factors and health. Epidemiology 2010; 21: 467-474.

30 Taniguchi H, Kondoh Y, Ebina M, et al. Pirfenidone Clinical Study Group in Japan. The clinical significance of $5 \%$ change in vital capacity in patients with idiopathic pulmonary fibrosis: extended analysis of the pirfenidone trial. Respir Res 2011; 12: 93.

31 Jones RL, Nzekwu MM. The effects of body mass index on lung volumes. Chest 2006; 130: 827-833.

32 Wang S, Sun X, Hsia TC, et al. The effects of body mass index on spirometry tests among adults in Xi'an, China. Medicine (Baltimore) 2017; 96: e6596.

33 Kondoh Y, Taniguchi H, Katsuta T, et al. Risk factors of acute exacerbation of idiopathic pulmonary fibrosis. Sarcoidosis Vasc Diffuse Lung Dis 2010; 27: 103-110.

34 Bhaskaran K, Dos-Santos-Silva I, Leon DA, et al. Association of BMI with overall and cause-specific mortality: a population-based cohort study of 3.6 million adults in the UK. Lancet Diabetes Endocrinol 2018; 6: $944-953$.

35 WHO Expert Consultation. Appropriate body-mass index for Asian populations and its implications for policy and intervention strategies. Lancet 2004; 363: 157-163.

36 Gallagher D, Heymsfield SB, Heo M, et al. Healthy percentage body fat ranges: an approach for developing guidelines based on body mass index. Am J Clin Nutr 2000; 72: 694-701.

37 Brown AW, Shlobin OA, Weir N, et al. Dynamic patient counseling: a novel concept in idiopathic pulmonary fibrosis. Chest 2012; 142: 1005-1010.

38 Yamana H, Moriwaki M, Horiguchi H, et al. Validity of diagnoses, procedures, and laboratory data in Japanese administrative data. J Epidemiol 2017; 27: 476-482. 